\title{
Estrutura populacional de capivaras na Estação Ecológica do Taim, Brasil, RS
}

\author{
Capybaras population structure in the Taim Ecological Station, Brazil, RS
}

\author{
Felipe Maia Garcias ${ }^{I}$ Alex Bager ${ }^{I I}$
}

RESUMO

Uma população de capivaras (Hydrochoerus hydrochaeris) foi monitorada na região limítrofe entre a Estação Ecológica do Taim e áreas de cultivo de arroz no extremo sul do Rio Grande do Sul, Brasil. O objetivo foi caracterizar a estrutura populacional, a densidade e os períodos reprodutivos da capivara na Estação Ecológica do Taim, RS, e em seu entorno. De agosto de 2003 a agosto de 2005, foram realizados 25 censos de contagem direta, em um transecto de 4.000 metros de comprimento, às margens da Lagoa Mangueira. Foi obtida a abundância de animais segundo seu

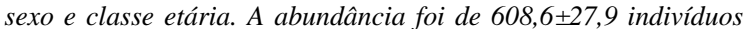

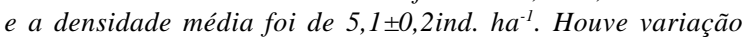
no número de animais do primeiro para o segundo ano de amostragem, sendo significativamente maior no último. Foram

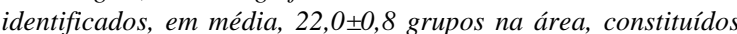
predominantemente de fêmeas adultas (47,5\%), seguidas de juvenis (24,8\%), filhotes (17\%) e machos adultos (7,7\%). A abundância de animais variou segundo a distribuição espacial, sendo maior no interior da Estação Ecológica do Taim (9,5ind. $\left.\mathrm{ha}^{-1}\right)$. Houve flutuações sazonais no recrutamento da população, tendo suas maiores densidades de filhotes no outono.

Palavras-chave: abundância, densidade, distribuição espacial, recrutamento.

\section{ABSTRACT}

A capybara population (Hydrochoerus hydrochaeris) was monitored in a borderline region between Taim Ecological Station and southernmost areas of rice cultivation located in the State of Rio Grande do Sul (RS, Brazil). The aim of this study was to characterize the structure, density, and reproductive periods of this population. A total of 25 censuses, obtained through direct count technique, were performed from August 2003 to August 2005. The samplings were performed in a 4000-meter length transect on the margins of Mangueira lagoon. The abundance was obtained considering the sex and age class of the animals. The abundance registered was of 608.6 \pm 27.9 individuals, and the average density was $5.1 \pm 0.2$ ind. $\mathrm{ha}^{-1}$. A variation in number of animals was observed between the first and the second year of sampling. This number was significantly higher in the last year. An average of $22.0 \pm 0.8$ groups was identified in the sampling area, including female adults (47.5\%), juveniles (24.8\%), young (17\%) and male adults (7.7\%). The abundance of animals varied according to spatial distribution, and its highest value (9.5ind. ha-1) was registered in Taim Ecological Station. Seasonal fluctuations in the population recruitment were also observed during this study. The highest densities of young were registered in autumn. Population structure was similar to those registered in different areas of distribution, but it has presented a high potential of population growth.

Key words: abundance, density, space distribution, recruitment.

\section{INTRODUÇÃO}

A capivara (Hydrochoerus hydrochaeris) é um herbívoro semiaquático, que se distribui em toda a América do Sul (com exceção do Chile e de áreas de elevada altitude) e se estende até o Panamá (YAI et al., 2008; EISENBERG \& REDFORD, 1999). É um animal gregário e social, que vive em bandos, podendo variar de 2 a 40 indivíduos (MOREIRA \& MACDONALD, 1997). Esses bandos apresentam uma rígida estrutura, havendo um macho adulto dominante que estabelece um harém com diversas fêmeas e seus filhotes (SCHALLER \& CRAWSHAW, 1981). A espécie ocupa habitats desde matas ciliares a savanas sazonalmente

Instituto Pró-Pampa, Pelotas, RS, Brasil.

IIDepartamento de Biologia, Universidade Federal de Lavras (UFLA), Grupo de Estudo em Ecologia de Estradas, 37200-000, Campus Universitário, Lavras, MG, Brasil. E-mail: abager@ufla.br. Autor para correspondência. 
inundáveis, manguezais e banhados. Os componentes compreendem corpos d'água, pastagens e matas, nos quais se protege de predadores e de fenômenos naturais (MOREIRA \& MACDONALD, 1997). Quando o número de predadores é reduzido, a espécie também pode ocupar áreas abertas, como campos e lagoas limpas (QUINTANA \& RABINOVICH, 1993).

A relação entre fauna selvagem e as atividades antrópicas tem sido amplamente discutida nos últimos anos (JAEGER, 2002; NAUGHTONTREVES et al., 2003). Danos por capivaras nas atividades agrícolas foram relatados por FERRAZ et al. (2003) e VERDADE \& FERRAZ (2006). No sul do Rio Grande do Sul, esse fato tem se verificado para as atividades agrícolas no entorno da Estação Ecológica do Taim, sobretudo naquelas áreas voltadas para a monocultura do arroz. Nesses ambientes, a capivara causa danos alimentando-se dos brotos de arroz e pisoteando a lavoura (GARCIAS \& BAGER, 2005).

A administração da área protegida tem se questionado acerca da necessidade da implantação de um programa de manejo da espécie. O objetivo deste trabalho foi caracterizar a estrutura populacional, a densidade e os períodos reprodutivos da capivara na Estação Ecológica do Taim, RS, e em seu entorno.

\section{MATERIAL E MÉTODOS}

\section{Caracterização da área em estudo}

Os censos foram realizados na região limítrofe entre a Estação Ecologica do Taim(ESEC Taim) e fazendas de cultivo de arroz. A ESEC Taim é uma área protegida federal, localizada na planície costeira do Estado do Rio Grande do Sul, Brasil, entre a Lagoa Mirim e o Oceano Atlântico. Compreende uma área de 33.818 hectares, sendo predominante a presença de áreas úmidas, sobretudo banhados, além de campos e dunas (GOMES et al., 1987). As áreas de matas são escassas, perfazendo $1 \%$ da sua área total. O clima é temperado e caracteriza-se pelo inverno frio e chuvoso e o verão quente e seco. A precipitação média anual é de $1.100 \mathrm{~mm}$ e a temperatura média é de $18^{\circ} \mathrm{C}$ (NIMER, 1989). As amostragens foram realizadas na margem oeste da Lagoa Mangueira (32S 50' 09"; 52W 38' 39").

\section{Levantamento dos dados}

As coletas de dados foram mensais, de agosto de 2003 a agosto de 2005, totalizando 25 períodos de coleta, realizadas sempre pelo mesmo observador. Cada amostragem foi compreendida por dois censos realizados em dias consecutivos e em diferentes horários, manhã e tarde, permitindo avaliar- se o efeito da hora na abundância dos animais. Durante o censo, todos os animais avistados foram contados. Sempre que possível, o indivíduo foi vinculado a um grupo e posicionado geograficamente por GPS. Cada indivíduo foi classificado segundo seu tamanho corporal em três classes etárias - adulto, juvenil e filhoteconforme proposto por VERDADE \& FERRAZ (2006). O sexo dos animais adultos foi registrado com base em seu dimorfismo sexual, constituído pela glândula sebácea proeminente entre as narinas e os olhos, presente exclusivamente nos machos. Observações noturnas auxiliaram no estabelecimento da largura da faixa de uso de habitat e em observações sobre o comportamento da espécie; contudo, não foram realizados censos noturnos devido à variação na eficiência das observações em relação àquelas executadas durante o dia, implicando mudanças na probabilidade de visualização dos animais. A largura de 300m, medida a partir da margem da lagoa, foi estabelecida por observações diretas, fezes e pegadas. Dos $4.000 \mathrm{~m}$ de comprimento monitorados, $2.000 \mathrm{~m}$ estavam dentro da ESEC Taim e 2.000m na sua área de entorno. As contagens dos indivíduos foram realizadas a distâncias que variaram de 1 a 50m dos animais, sendo executadas a olho nu ou com binóculo.

A distribuição espacial foi avaliada considerando-se a variação da densidade ecológica em cada segmento de $500 \mathrm{~m}$ do transecto de amostragem. O primeiro trecho $(0-500 \mathrm{~m})$ foi considerado no extremo norte da área (dentro da ESEC Taim) e o último (3500$4000 \mathrm{~m}$ ) no extremo sul, fora da área da ESEC Taim (Figura 1). Os grupos de capivaras foram distribuídos em cada segmento utilizando-se a posição geográfica coletada com GPS. Foram realizadas caracterizações do ambiente aquático e terrestre visando-se a estabelecer classes de uso e vegetação em cada um dos trechos descritos. Em ambiente terrestre, foram utilizadas as classes plantação de arroz, pastagem e urbanizada e, em ambientes aquáticos, consideraram-se as classes com e sem macrófitas. O ambiente terrestre foi caracterizado pela presença de pastagem na porção mais próxima à margem da lagoa e por plantação de arroz a partir de $100 \mathrm{~m}$ da margem. As únicas variações em tal padrão foram a ausência de atividade agrícola no primeiro quadrante $(0-500 \mathrm{~m})$ e a presença de construções e estrada no quadrante localizado entre 2.000 e $2.500 \mathrm{~m}$. No ambiente aquático, de 0 a $2.500 \mathrm{~m}$, não houve presença significativa de macrófitas aquáticas. Já nos três últimos quadrantes (2.500-4.000m), foi constatada a presença de espécies flutuantes e enraizadas (p.ex.: Pistia stratiotes, Espindela sp, Biolens sp, Scirpus californicus).

Ciência Rural, v.39, n.8, nov, 2009. 


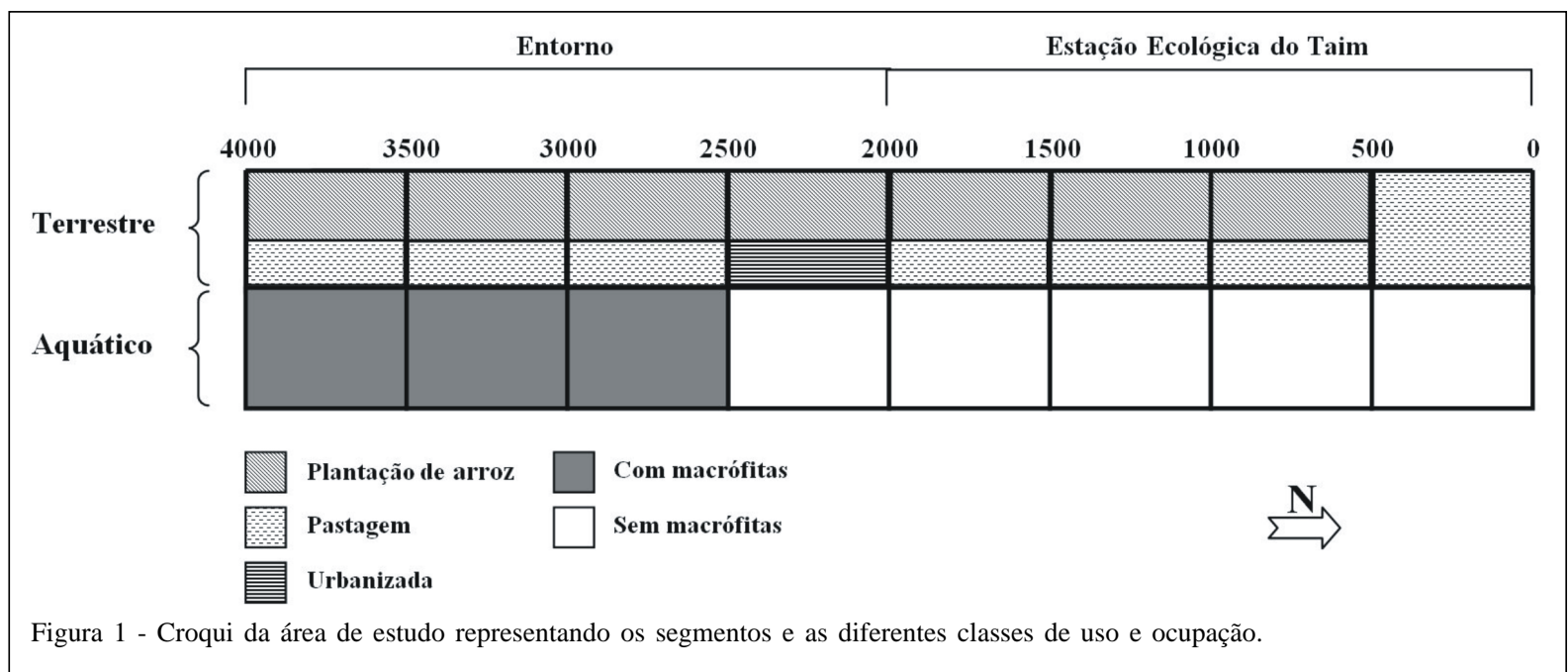

Análise de dados

A normalidade dos dados foi analisada com Shapiro Wilk's e a homocedasticidade com o teste de Levene. Dados normais foram comparados utilizandose ANOVA, enquanto que os não paramétricos foram comparados com Kruskal-Wallis. O número de animais contados no período da manhã foram comparados com os da tarde utilizando-se Análise de Variância (ANOVA) para amostras repetidas (SOKAL \& ROHLF, 1987). Foram utilizados o índice de abundância e o de densidade ecológica, ambos baseados na contagem direta de cada indivíduo (VERDADE \& FERRAZ, 2006). A densidade foi calculada considerando-se uma área de 120 ha (4.000 $\mathrm{m} \times 300 \mathrm{~m})$.

A densidade ecológica de adultos (machos e fêmeas) foi comparada em dois períodos de amostragens anuais, Ago/03-Jul/04 e Ago/04-Jul/05. Tanto as análises espaciais como as temporais utilizaram o teste de Kruskal-Wallis, e as comparações de médias foram realizadas com o teste de Tukey (ZAR, 1999). As médias, sempre que conveniente, foram apresentadas com seu erro padrão associado (Média \pm Erro Padrão). Todas as análises consideraram uma significância de 0,05 .

\section{RESULTADOS}

O número de animais observados em diferentes horas do dia não apresentou diferença significativa $\left(\mathrm{F}_{1,24} 1,697 ; \mathrm{P}=0,205\right)$. Dessa forma, optouse pelo agrupamento dos dados calculando-se a média mensal. O número de grupos variou entre 15 e 29 (22 $\pm 0,8 ; \mathrm{N}=1.101)$. O número de indivíduos por grupo

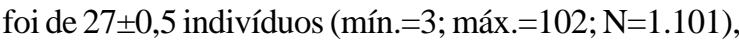
predominando aqueles com 11 indivíduos. Os machos corresponderam a $7,7 \%$ da estrutura dos grupos, enquanto que as fêmeas corresponderam a $47,5 \%$, os juvenis a $24,8 \%$ e os a filhotes $17 \%$. Outros $3 \%$ foram de adultos cujo sexo não foi identificado. A razão sexual foi de 6,6 60,1 fêmeas por macho (mín.=0,5; máx.=37; $\mathrm{N}=986$ ), com moda nos grupos contendo cinco fêmeas.

O índice de abundância médio da população foi de 608,6 627,9 indivíduos. Já a densidade média total foi de 5,1 \pm 0 ,2ind. ha ${ }^{-1}$ (Tabela 1 ). A partir de março de 2004, observou-se tendência de aumento na densidade média da população total, apesar de decréscimos em períodos específicos (Mai/2004; Jan e Mar/05) (Figura 2). A densidade da população no primeiro ano de amostragem foi $4,1 \pm 0$,2ind. ha ${ }^{-1}$ e no segundo ano foi de 5,9 $\pm 0,2$ ind.ha $^{-1}$, sendo significativamente maior no último ano $\left(\mathrm{F}_{1,11} 42,28 ; \mathrm{P}<0,001\right)$. Tanto os adultos quantos os juvenis apresentaram diferença significativa na densidade entre o primeiro e o segundo ano (Adultos: $\mathrm{F}_{1,11}$ 33,33; $\mathrm{P}<0,001$; Juvenis: $\mathrm{F}_{1,11}$ 66,62; $\mathrm{P}<0,001)$. Filhotes foram observados em todas as estações do ano $\left(0,89 i n d\right.$. ha $\left.{ }^{-1}\right)$, tendo ocorrido um aumento da sua densidade no outono de 2004 (1,5ind. $\left.\mathrm{ha}^{-1}\right)$, não sendo o mesmo observado no outono de 2005.

A densidade da população de capivaras variou espacialmente, sendo significativamente maior na ESEC Taim ( $\bar{x}=7$ ind. ha-1 $)$ do que no seu entorno ( $=1$,7ind. ha $\left.{ }^{-1}\right)\left(\mathrm{H}_{(7, \mathrm{~N}=200)}=151,333 \mathrm{P}<0,001\right)$. O teste de Tukey revelou um gradiente de quatro diferentes densidades (Tabela 2). A combinação das características ambientais e da densidade de capivaras demonstra que as áreas com menor densidade estiveram relacionadas aos quadrantes com presença de macrófitas aquáticas. As densidades intermediárias foram encontradas nos quadrantes com ausência de macrófitas, mas com maior atividade antrópica, e a maior concentração de animais foi encontrada dentro da ESEC Taim.

Ciência Rural, v.39, n.8, nov, 2009. 
Tabela 1 - Abundância e densidade de capivaras (Hydrochaerus hydrochaeris) na Estação Ecológica do Taim, RS, Brasil.

\begin{tabular}{lcc}
\hline & $\begin{array}{c}\text { Ind. de Abundância } \\
\text { (ind.) }\end{array}$ & $\begin{array}{c}\text { Densidade } \\
\text { (ind. ha }^{-1} \text { ) }\end{array}$ \\
\hline Machos & $47,1 \pm 2,5$ & $0,4 \pm 0,0$ \\
Fêmeas & $285,2 \pm 12,4$ & $2,4 \pm 0,1$ \\
Juvenis & $153,6 \pm 11,9$ & $1,3 \pm 0,1$ \\
Filhotes & $106,4 \pm 10,7$ & $0,9 \pm 0,1$ \\
A.N.I.* & $16,8 \pm 2,2$ & $0,1 \pm 0,0$ \\
Total & $608,6 \pm 27,9$ & $5,1 \pm 0,2$ \\
\hline
\end{tabular}

*A.N.I. - Adultos não identificados.

**Valores são seguidos pelo erro padrão da média.

O carcará (Caracara plancus) predou a espécie alimentando-se de filhotes e adultos doentes. A predação de filhotes ocorreu com maior freqüência. Adultos doentes, com pouca mobilidade, foram atacados na sua porção dorsal e eventualmente nos olhos.

\section{DISCUSSÃO}

A densidade de 587ind. $\mathrm{km}^{-2}$ foi a maior já descrita para a espécie, independentemente do ambiente considerado (MACDONALD, 1981; ALHO \& RONDON, 1987; ALHO et al., 1987). A maior densidade para o Brasil foi citada por VERDADE \& FERRAZ (2006), que observaram a densidade de 124ind. $\mathrm{km}^{-2}$ e estimaram uma capacidade-suporte de 195ind. $\mathrm{km}^{-2}$. Desconhecem-se os fatores que determinaram essa elevada densidade, mas observações em outras áreas da ESEC Taim mostraram uma grande heterogeneidade espacial na distribuição da espécie na Unidade de Conservação (BAGER, 2009 - informe verbal). Essa variação deve estar condicionada a fatores de disponibilidade de recurso alimentar (natural, pastagem e agricultura de arroz), pela aparente conscientização de alguns dos proprietários de terras no entorno da Estação e pela presença contínua de fiscais do IBAMA. FERRAZ et al. (2006) associaram a presença de capivaras a áreas planas, com pastagem e cultura de cana. Na ESEC Taim, os animais utilizavam a pastagem durante o dia e a plantação de arroz durante a noite. Esse comportamento possivelmente está relacionado à distância entre a plantação e a água ( 100m), o que os torna vulneráveis à predação em períodos de maior luminosidade.

Ausência de variação em censos realizados nos períodos de manhã e tarde é frequente em populações de capivaras. MACDONALD (1981) verificou semelhança no número de animais observados em campo; entretanto, relatou que, no período da manhã, predomina o repouso, enquanto que à tarde a principal atividade é o forrageamento. LORD (1991) constatou pouca variação diurna na atividade das capivaras. SALAS et al. (2004) afirmaram que a espécie nunca se desloca mais de $500 \mathrm{~m}$ da margem

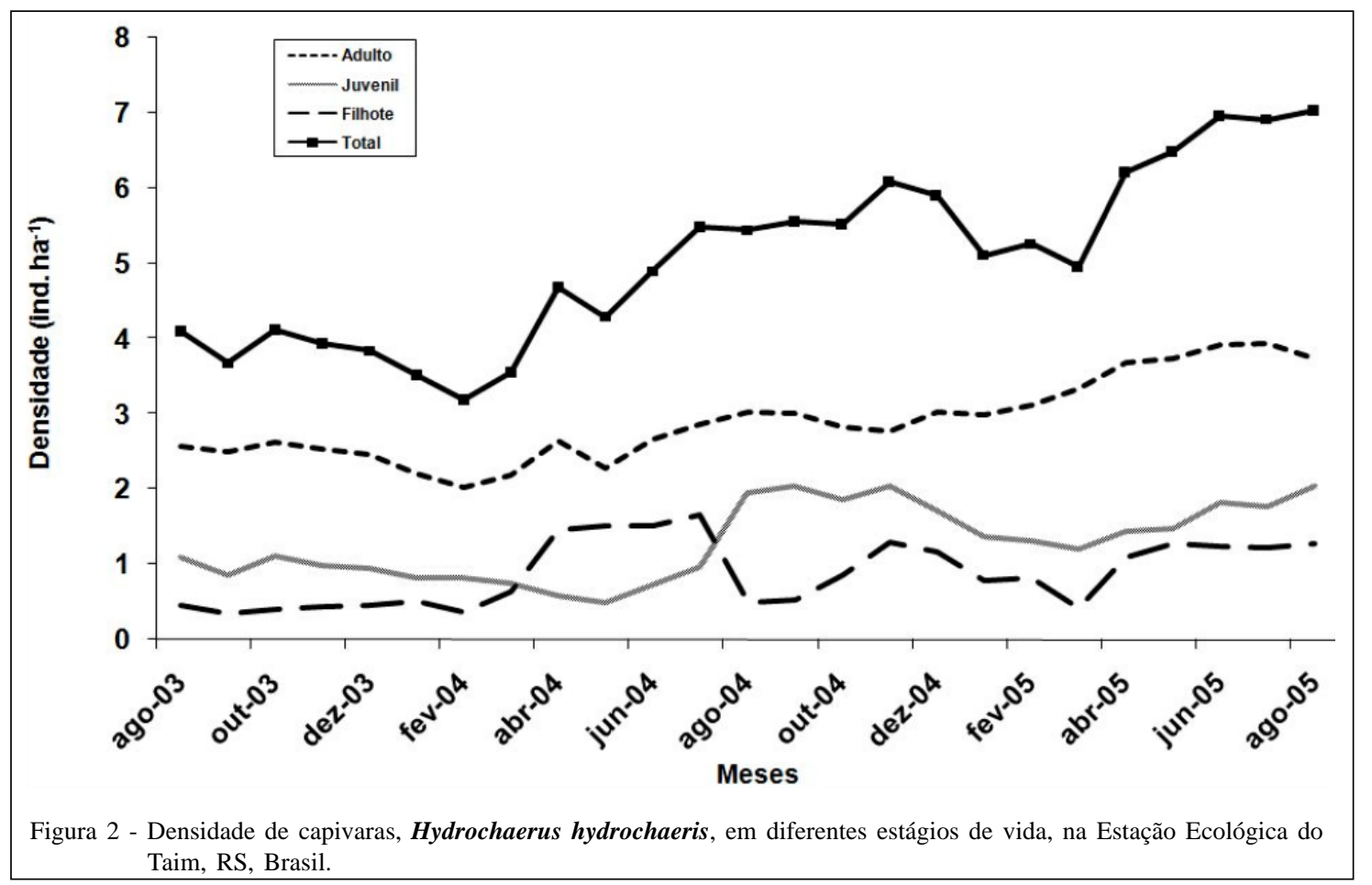

Ciência Rural, v.39, n.8, nov, 2009. 
Tabela 2 - Densidade em relação à distribuição espacial nos segmentos de $500 \mathrm{~m}$ da população de capivaras (Hydrochaerus hydrochaeris) na Estação Ecológica do Taim, RS, e na sua área de entorno.

\begin{tabular}{lccccc}
\hline & Média & EP & Mín. & Máx. & N \\
\hline $0^{\text {a }}$ & 9,5 & 0,7 & 3,6 & 16,5 & 25 \\
$500^{\mathrm{b}}$ & 7,1 & 0,5 & 2,9 & 12,0 & 25 \\
$1000^{\mathrm{b}}$ & 7,6 & 0,4 & 4,1 & 11,2 & 25 \\
$1500^{\mathrm{b}}$ & 7,6 & 0,4 & 5,3 & 11,8 & 25 \\
$2000^{\mathrm{c}}$ & 3,3 & 0,3 & 0,9 & 5,7 & 25 \\
$2500^{\mathrm{c}}$ & 4,6 & 0,4 & 0,7 & 8,5 & 25 \\
$3000^{\mathrm{d}}$ & 0,6 & 0,1 & 0,0 & 2,9 & 25 \\
$3500^{\text {d }}$ & 0,1 & 0,1 & 0,0 & 1,1 & 25 \\
\hline
\end{tabular}

Letras iguais identificam segmentos com densidades iguais $(\mathrm{P}>0,05)$.

d’água. Observações não sistemáticas demonstraram que, no período noturno, a espécie se distancia mais da margem da lagoa, apresentando deslocamentos máximos de 300m, provavelmente em decorrência do aumento da atividade humana pelas moradias e do movimento de maquinário nas áreas mais distantes da lagoa.

O número de indivíduos por grupo foi o maior já relatado para a espécie, podendo ultrapassar 100 indivíduos. Esse fato pode ter ocorrido em conseqüência de fatores ambientais, tais como épocas de intensa precipitação e consequente redução das áreas de pastagem, onde dois grupos próximos foram classificados como um único grupo. A variação do número de grupos na ESEC Taim parece corroborar essa possibilidade. JORGENSON (1986) também observou a fusão temporária de grupos, tendo encontrado valores entre 7 e 57 animais. Apesar da amplitude elevada no tamanho dos grupos, a maioria dos grupos da ESEC Taim foi composta por 11 indivíduos, o que se assemelhou aos resultados de HERRERA \& MACDONALD (1987).

A proporção entre machos e fêmeas (1 macho/6,6 fêmeas) foi inferior àquela descrita por HERRARA \& MACDONALD (1987) (1 macho/1,7 fêmeas) e por HERRERA \& MACDONALD (1993) (1 macho/2,8 fêmeas). Contudo, a proporção de machos aumentou durante o período de estudo. A estrutura encontrada na ESEC Taim foi semelhante àquela descrita por ALHO \& RONDON (1987), havendo diferença somente na proporção de juvenis, maior no presente trabalho. Trabalhos anteriores também evidenciam que a reprodução ocorre o ano todo, sendo registrados períodos de maior recrutamento em épocas específicas. O maior recrutamento no outono corrobora os dados de VERDADE \& FERRAZ (2006) para o interior de São Paulo, Brasil.
ALHO et al. (1987) relataram que o habitat ideal para a capivara é composto por floresta, pasto e água. A ESEC Taim possui poucas áreas florestadas e observações não sistemáticas indicam que as densidades nessas áreas são menores que as constatadas nos demais ambientes (BAGER, 2009 informe verbal). Nossos dados são congruentes com os descritos por FERRAZ et al. (2006), sendo que a combinação de áreas planas e pastagens/agricultura são características fundamentais para a ocorrência de capivara. Os trechos com menor densidade de animais foram aqueles com grande concentração de macrófitas enraizadas e flutuantes. Essas áreas são paralelas à margem da lagoa, tendo entre 30 e 100m de largura, e atuam como uma barreira ao deslocamento dos animais entre as áreas de pastagem e a água. Áreas com presença de estradas de acesso às plantações tiveram menor densidade que áreas sem trânsito de veículos. A população apresentou preferência por áreas onde a presença humana seja minimizada e que haja o acesso direto a água. Nas áreas de maior densidade, a única proteção contra predadores é a lagoa Mangueira, não havendo vegetação arbustiva ou arbórea.

Apesar de ocorrência de vários predadores potenciais como lontras (Lontra longicaudis), canídeos (Cerdocyon thous e Lycalopex gymnocercus) e gatos-do-mato-grandes (Leopardus geoffroyi), somente foi constada predação de capivara por Caracara plancus. Essa espécie é um predador generalista, que atua sobre diversos grupos de vertebrados (SICK, 1997; PETRACCI \& BASANTA, 2002; BRANCO \& FRACASSO, 2005). JORGENSON (1986) e LORD (1994) também citam a predação de jovens capivaras pela espécie. Já TOMAZZONI et al. (2005) descrevem associações entre as duas espécies, nas quais $\boldsymbol{C}$. plancus se alimentou de ectoparasitas encontrados em $\boldsymbol{H}$. hydrochaeris.

\section{CONCLUSÕES}

A estrutura da população de Hydrochoerus hydrochaeris na ESEC Taim é semelhante às descritas para outras áreas de ocorrência da espécie, mas possui grande potencial de continuar aumentando. A espécie apresentou incremento de $43 \%$ na densidade de indivíduos entre o primeiro e o segundo ano de amostragem; adicionalmente, a espécie realiza recrutamento durante todo o ano, sendo que a população descrita apresenta razão sexual baixa quando comparada a outras populações. Isso supõe alto potencial de incorporação de novos machos, seja de outras localidades ou pela divisão dos grandes grupos em haréns menores. Outros indicativos desse 
potencial de crescimento populacional são as baixas taxas de predação e a grande disponibilidade de recursos oriundos do banhado e das áreas de plantação. Sugere-se que futuros trabalhos busquem a análise espaço-temporal da constituição dos grupos, considerando aspectos de deslocamento, dispersão e comportamento, assim como estimem a capacidadesuporte do ambiente.

\section{INFORME VERBAL}

BAGER, A. Universidade Federal de Lavras, Departamento de Biologia, Setor de Ecologia, Campus Universitário, C.P. 3037, Lavras, MG, email: abager@ufla.br.

\section{REFERÊNCIAS}

ALHO, C.J.R. et al. Ecologia de capivaras (Hydrochaeris hydrochaeris, Rodentia) do Pantanal: I, hábitats, densidades e tamanho de grupo. Revista Brasileira de Biologia, v.47, p.87-97, 1987.

ALHO, C.J.R.; RONDON, N.L. Habitats, population densities, and social structure of Capybaras (Hydrochaeris hydrochaeris, Rodentia) in the Pantanal, Brazil. Revista Brasileira de Zoologia, v.4, p.139-149, 1987.

BRANCO, J.O.; FRACASSO, H.A.A. Reprodução de Nycticorax nycticorax (Linnaeus) no litoral de Santa Catarina, Brasil. Revista Brasileira de Zoologia, v.22, p.424-429, 2005. Disponível em: <http://www.scielo.br/scielo.php?pid=S0101$81752005000200018 \&$ script $=$ sci_arttext \&tlng=pt $>$. Acesso em: 16 jul. 2009. doi: 10.1590/S0101-81752005000200018.

EISENBERG, J.F.; REDFORD, K.H. Mammals of the neotropics: the central neotropics. Chicago: University of Chicago, 1999. 609p.

FERRAZ, K.M.P.M.B. et al. Damage caused by capybaras in a corn field. Scientia Agricola, v.60, p.191-194, 2003. Disponível em: <http://www.scielo.br/scielo.php?pid=S010390162003000100029\&script=sci_arttext $>$. Acesso em: 16 jul. 2009. doi: 10.1590/S0103-90162003000100029.

FERRAZ, K.M.P.M.B. et al. Capybara (Hydrochoerus hydrochaeris) distribution in agroecosystems: a crossscale habitat analysis. Journal of Biogeography, v.34, p.223-230, 2006. Disponível em: <http://www3.interscience.wiley.com/cgi-bin/ fulltext/117963819/HTMLSTART>. Acesso em: 16 jul. 2009. doi: $10.1111 /$ j.1365-2699.2006.01568.x.

GARCIAS, F.M.; BAGER, A. A problemática da interação homem-animal selvagem no entorno da Estação Ecológica do Taim-RS. In: SIMPÓSIO DE ÁREAS PROTEGIDAS, 3., 2005, Pelotas, RS. Anais... Pelotas: Alex Bager, 2005. 638p. p.418424.

GOMES, A. et al. Estudos ecodinâmico da Estação Ecológica do Taim e seus arredores. Porto Alegre: UFRGS, 1987. 84p.

HERRERA, E.A.; MACDONALD, D.W. Group stability and the structure of a capybara population. Symposium of the Zoological Society of London, v.58, p.115-130, 1987.
HERRERA, E.A.; MACDONALD, D.W. Aggression, dominance, and mating success among capybara males (Hydrochaeris hydrochaeris). Behavioral Ecology, v.4, p.114119, 1993. Disponível em: <http://beheco.oxfordjournals.org/ cgi/content/abstract/4/2/114>. Acesso em: 16 jul. 2009. doi:10.1093/beheco/4.2.114.

JAEGER, P. High-level pan-european conference on agriculture and biodiversity: towards integrating biological and landscape diversity for sustainable agriculture in Europe, 2002. Acessado em 5 fev. 2009. Online. Disponível em: <http://www.lcie.org/Docs/COE/CoE\%20Jaeger\%20Conflic ts\%20between\%20wildlife\%20and\%20agriculture.pdf $>$.

JORGENSON, J.P. Notes on the ecology and behavior of capybaras in northeastern Colombia. Vida Silvestre Neotropical, v.1, p.31-40, 1986.

LORD, R.D. Twenty-four-hour activity and coprofagy by capybaras (Hydrochaeris hydrochaeris). Studies on Neotropical Fauna and Environment. v.26, p.113-120, 1991. Disponível em: <http://www.informaworld.com/smpp/ content $\sim \mathrm{db}=\mathrm{all} \sim \mathrm{content}=\mathrm{a} 905702820>$. Acesso em: $16 \mathrm{jul}$. 2009. doi: 10.1080/01650529109360840.

LORD, R.D. A descriptive account of capybara behaviour. Studies on Neotropical Fauna and Environment, v.29, p.11-22, 1994. Disponível em: <http://www.informaworld.com/ smpp/content $\sim \mathrm{db}=\mathrm{all} \sim \mathrm{content}=\mathrm{a} 905702618>$. Acesso em: 16 jul. 2009. doi: 10.1080/01650529409360912.

MACDONALD, D.W. Dwindling resources and the social behaviour of capybaras, (Hydrochoerus hydrochaeris) (Mammalia). Journal of Zoology. v.194, p.371-391, 1981.

MOREIRA, J.R.; MACDONALD, D.W. Técnicas de manejo de capivaras e outros grandes roedores na Amazônia. In: VALLADARES-PADUA, C. et al. (Eds.). Manejo e conservação de vida silvestre no Brasil. Belém: Sociedade Civil Mamiraúa, 1997. p.186-213.

NAUGHTON-TREVES, L. et al. Wildlife survival beyond park boundaries: the impact of slash-and-burn agriculture and hunting on mammals in Tambopata, Peru. Conservation Biology, v.17, p.1106-1117, 2003. Disponível em: <http:// www3.interscience.wiley.com/cgi-bin/fulltext/118885643/ HTMLSTART>. Acesso em: 16 jul. 2009. doi: 10.1046/j.15231739.2003.02045.x.

NIMER, E. Climatologia do Brasil. Rio de Janeiro: IBGESUPREN, 1989. 421p.

PETRACCI, P.F.; BASANTA, D. Efectos positivos de la nidificación del macá común (Rollandia rolland) en una colonia de caracoleros (Rostrhamus sociabilis). Ornitologia Neotropical, v.13, p.113-119, 2002. Disponível em: <http:/ /elibrary.unm.edu/sora/ON/v013n02//p0113-p0120.pdf. Acesso em: 16 jul. 2009.

QUINTANA, R.D.; RABINOVICH, J.E. Assessment of capybara (Hydrochoerus hydrochaeris) populations in the wetlands of Corrientes, Argentina. Wetlands Ecology and Management, v.2, p.223-230, 1993. Disponível em: <http:/ /www.springerlink.com/content/t25124358lk27734/ ?p=f21172140a44419e921a36050b3ca55a\&pi=5>. Acesso em: 16 jul. 2009. doi: 10.1007/BF00188156. 
SALAS, V. et al. Methods for capturing and marking wild capybaras in Venezuela. Wildlife Society Bulletin,v.32, p.202-208, 2004. Disponível em: <http://www.bioone.org/doi/full/10.2193/00917648\%282004\%2932\%5B202\%3AMFCAMW\%5D2.0.CO\%3B2>. Acesso em: 16 jul. 2009. doi: 10.2193/00917648(2004)32[202:MFCAMW]2.0.CO;2.

SCHALLER, G.B.; CRAWSHAW, P.G. Social organization in a capybara population. Säugetierkundliche Mitteilungen, v.29, p.3-16,1981.

SICK, H. Ornitologia brasileira. Rio de Janeiro: Nova Fronteira, 1997. 912p.

SOKAL, R.R.; ROHLF, F.J. Introduction to biostatistics. New York: W. H. Freeman, 1987. 363p.

TOMAZZONI, A.C. et al. Feeding association between capybaras Hydrochoerus hydrochaeris (Linnaeus) (Mammalia, Hydrochaeridae) and birds in the Lami Biological Reserve, Porto
Alegre, Rio Grande do Sul, Brazil. Revista Brasileira de Zoologia, v.22, p.712-716. 2005. Disponível em: <http:// www.scielo.br/pdf/rbzool/v22n3/26194.pdf $>$. Acesso em: 16 jul. 2009. doi: 10.1590/S0101-81752005000300031.

VERDADE, L.M.; FERRAZ, K.M.P.M.B. Capybaras in an anthropogenic habitat in southeastern Brazil. Brazilian Journal of Biology, v.66, p.371-378, 2006. Disponível em: <http://www.scielo.br/pdf/bjb/v66n1b/a19v661b.pdf>. Acesso em: 16 jul. 2009. doi: 10.1590/S1519-69842006000200019.

YAI, L.E.O. et al. Isolation of toxoplasma gondii from capybaras (Hydrochaeris hydrochaeris) from São Paulo State, Brazil. Journal of Parasitology, v.94, p.1060-1063, 2008. Disponível em: <http://www.scielo.br/pdf/bjb/v66n1b/ a19v661b.pdf>. Acesso em: 16 jul. 2009. doi: 10.1645/GE1548.1 .

ZAR, J.H. Biostatistical Analysis. Upper Saddle River: Prentice-Hall, 1999. 663p. 